\title{
Inhibition of Porphyromonas Gingivalis Gingipain Activity by Prenylated Flavonoid, Sanggenol A.
}

\author{
Grant King1, Margaret Jefferson ${ }^{2}$, Edwin L Thomas ${ }^{2}$, Sidney H Stein ${ }^{1}$, John Hamed Jefferia ${ }^{1}$ and Jegdish P \\ $\mathrm{Babu}^{2 *}$
}

${ }^{1}$ Department of Periodontology, University of Tennessee Health Science Center, USA

${ }^{2}$ Department of Bioscience Research, University of Tennessee Health Science Center, USA

*Corresponding author: Jegdish P Babu, Department of Bio science Research, College of Dentistry, University of Tennessee Health Science Center, USA.
Received Date: July 12, 2019

Published Date: July 22, 2019

\section{Abstract}

Background and objective: Porphyromonas gingivalis (Pg), is an important periodontal pathogen known to possess various virulence factors such as the extracellular gingipain proteases. These play a role in destruction of periodontal tissue. Naturally occurring polyphenols were shown to play a role in preventing bacterial pathogenicity by neutralizing proteolytic enzymes. We sought to examine the role of a prenylated flavonoid, Sanggenol A, on neutralization of Pg gingipain proteolytic activity.

Materials and methods: The effect of Sanggenol A on the secreted and cell-associated gingipain activity of Pg was measured using fluorogenic substrates, and the resulting fluorescence was measured by fluorescence reader.

Results: The results revealed an inverse correlation between the concentration of Sanggenol A and both secreted and Pg cell-associated gingipain activity. The activity of R gingipain (Rgp) was found to be significantly more susceptible to Sanggenol A inhibition than the K gingipain (Kgp) $(\mathrm{P}=0.03)$. Bacteria grown in the presence of Sanggenol A also exhibited reduced gingipain proteolytic activity.

Conclusion: We conclude that the natural flavonoid, such as Sanggenol A is effective in neutralizing the proteolytic activity of the periodontal pathogen. The results suggest that this flavonoid may play a useful role in preventing or reducing the periodontal tissue destruction induced by Pg.

Keywords: Sanggenol A; Porphyromonas gingivalis; Gingipains; Protease

\section{Introduction}

The gram-negative oral anaerobe Porphyromonas gingivalis (Pg) is a major etiological pathogen in the inception of adult periodontitis. The disease is characterized by gingival inflammation and bleeding, soft tissue attachment loss, and alveolar bone loss [1,2]. Even in low abundance, $P$. gingivalis is capable of modulating the host immune response, guiding biofilm synergy and growth, impacting biofilm gene expression, and increasing the pathogenicity of the collective biofilm via an abundance of virulence factors [3-8]. P. gingivalis has also been reported to selectively activate the Th17 pathway and promote IL-17 secretion, increased levels of which have been associated with periodontitis [9]. P. gingivalis has been known to secrete an abundance of proteases, known as "gingipains", which are a specific group of trypsin-like cysteine proteases responsible for $\sim 85 \%$ of $P$. gingivalis proteolytic activity and $99 \%$ of the "trypsinlike" activity [10-14]. Gingipains are classified depending on the polypeptide bonds they cleave, either as arginine, encoded by RgpA and RgpB genes or lysine, by Kgp gene gingipains. Both enzymes can occur as cell membrane-bound or as secreted forms $[15,16]$. In addition, gingipains provide nutrients (via protein degradation), and contributes to adhesion and tissue invasion, destruction of host tissue, platelet activation, RBC agglutination, hemolysis, and dysregulation of the host immune response $[14,17,18]$. Due to their significant role in adult periodontitis, inhibition of gingipain protease activity would be a potential therapeutic approach for diminishing $P$. gingivalis virulence and an alternative to antibiotic therapy for management of periodontal disease.

Several classes of gingipain inhibitors have been identified, including plant-derived aromatic compounds called polyphenols, more specifically flavonoids [19]. Polyphenols, often referred to as flavonoids, are touted for a variety of biologic functions, most 
notably for their antioxidant, anti-inflammatory, anti-cancer, and anti-microbial properties [20]. Kariu et al. reported that a flavonoid, Limonianin, inhibited biofilm growth and gingipain activity of $P$. gingivalis [21]. Similarly, a number of prenylated flavonoids derived from the Epimedium species also inhibited gingipain activity and hindered P. gingivalis growth and biofilm formation [21]. Morus alba, or mulberry tree, has traditionally been used for medicinal purposes due to its anti-inflammatory, hypoglycemic, anti-bacterial, and anti-viral properties [22-26]. Park et al. demonstrated that an extract from Morus alba root bark, known as Kuwanon G, inhibited the growth of the oral pathogens, Streptococcus mutans, Streptococcus sobrinus, Streptococcus sanguis, and P. gingivalis [27]. Recently, Sanggenol A, another extract from Morus alba root bark, was also shown to inhibit influenza A virus and neuraminidase activity of S. pneumonia [28], but nothing is known of the effect of Sanggenol A on P. gingivalis protease activity. The purpose of the present study was to investigate the effect of Sanggenol A on the activity of $P$. gingivalis cell bound gingipain activity and activity released into the cell culture medium.

\section{Methods}

\section{Reagents}

Sanggenol A was purchased from Wuhan Chem Faces Biochemical Co., Ltd. Wuhan, Hubei, China. Dimethyl sulfoxide (DMSO), menadione, phenylmethanesulfonyl fluoride (PMSF), Tosyl-L-lysine chloromethyl ketone hydrochloride (TLCK), and Benzoyl-R-AMC were obtained from Sigma-Aldrich, St. Louis, MO. Boc-VLK-AMC was purchased from Bachem Americas, Torrance, CA.

\section{Preparation of sanggenol A}

A 0.1 M stock solution of Sanggenol A was prepared in $26 \%$ DMSO, and the stock was subsequently diluted (1 to $10,000 \mu \mathrm{M}$ ) in Dulbecco's phosphate buffered saline (DPBS) free of calcium and magnesium (Life Technologies, Carlsbad, CA).

\section{Bacterial strain and culture conditions}

P. gingivalis strain 33277 was obtained from the American Type Culture Collection (Manassas, VA). The bacteria were grown for 24 $\mathrm{h}$ at $37^{\circ} \mathrm{C}$ under anaerobic conditions in trypticase soy broth (TSB) supplemented with $1 \%$ yeast extract (Becton, Dickinson \& Co., Spark, MD) along with hemin $(5 \mu \mathrm{g} / \mathrm{mL})$ and menadione $(1 \mu \mathrm{g} / \mathrm{mL})$. The purity and sterility of the culture was confirmed by plating the bacterial suspension on $5 \%$ sheep blood agar plates (Becton, Dickinson \& Co.). Additionally, serial dilutions of the cultures were made in sterile DPBS and plated on blood agar plates to determine the number of colony-forming units (CFU) per ml.

\section{Preparation of $\boldsymbol{P}$. gingivalis cell-associated and secreted gingipains in conditioned media}

Bacterial cultures after 24 hours of growth were collected and centrifuged to separate the cells and the culture supernatants. The supernatants were filtered through $0.2 \mu \mathrm{m}$ filters. The filtered supernatant was supplemented with $0.2 \mathrm{mM}$ PMSF to block serine protease activity. To measure cell-associated gingipain activity, bacterial cells grown for $24 \mathrm{~h}$ as described above, were suspended in sterile DPBS supplemented with PMSF to an optical density of 4.0 at $600 \mathrm{~nm}$ (approximately equivalent to $4 \times 109 \mathrm{CFU} / \mathrm{mL}$ ). Aliquots of the culture media and bacterial suspensions were kept frozen at $-80^{\circ} \mathrm{C}$. Activity of gingipain Rgp and Kgp in cell-free bacterial supernatants and cell-associated was determined by using $10 \mathrm{mM}$ Benzoyl-R-AMC and Boc-VLK-A substrates, respectively. Stock solutions $(20 \mathrm{mM})$ of these substrates were prepared in DMSO and stored at $-80^{\circ} \mathrm{C}$ in aliquots of $50 \mu \mathrm{L}$. Substrate stock solutions were diluted to $2 \mathrm{mM}$ with DPBS prior to their use in the assays.

\section{Assay for gingipain activity and effect of the inhibitor, sanggenol A}

The activity of cell-associated gingipain was measured according to the methods described by [30]. Briefly, we incubated $20 \mu \mathrm{l}$ of the stock bacterial cell suspension diluted 50fold in DPBS containing $1 \mathrm{mM}$ L-cysteine to ensure the reduced state (active form) of gingipains for $10 \mathrm{~min}$ at $37^{\circ} \mathrm{C}$. The cell-free culture media $(20 \mu \mathrm{l})$ after two-fold dilution in DPBS with $1 \mathrm{mM}$ L-cysteine was incubated for 10 minutes at $37^{\circ} \mathrm{C}$ in a black 96-well plate (Fischer Scientific). Following this initial incubation, the substrate, benzoylR-AMC or Boc-VLK-AMC, was added to give $0.2 \mathrm{mM}$ in a final volume of $100 \mu \mathrm{l}$. The reaction mixture was incubated further for $15 \mathrm{~min}$ at $37^{\circ} \mathrm{C}$. The reaction was stopped by adding $50 \mu \mathrm{L}$ of $1.5 \mathrm{mM}$ TLCK. The hydrolysis of the substrates by gingipains was measured by fluorescence emission which was measured by a fluorescence reader (Spectra Max Gemini EM, Molecular devices, Sunnyvale, CA) with excitation at $365 \mathrm{~nm}$ and emission at $460 \mathrm{~nm}$.

To investigate the effect of the inhibitor, Sanggenol A stock solution was diluted in DPBS $(1-10,000 \mathrm{mM})$ and a $10 \mu \mathrm{L}$ aliquot of diluted Sanggenol A was added to the above reaction mixtures. As a control, we added DPBS instead of Sanggenol A and incubated with the protease substrates as described above. To determine the effect of Sanggenol A on the gingipain activity of Pg, bacteria were cultured as described previously in the media supplemented with 10,30 or $100 \mu \mathrm{M}$ Sanggenol A. Cells and media were collected, diluted and incubated with the two protease substrates and the cell-associated and secreted gingipain activities were measured.

\section{Results}

\section{Demonstration of gingipain activity in cells and media}

Gingipain activity was measured in resuspended cells and the cell-free culture media. Activity of both Rgp and Kgp was higher in the cell suspensions than in the media, and the total Rgp activity was 4.6 times greater than the total Kgp activity ( $\mathrm{P}=0.001)$. Rgp activity in cell suspensions and culture media was 1,318,774 and $91,701 \mathrm{RFU} / \mathrm{min} / \mathrm{mL}$ of culture, respectively, and Kgp activity was 289,494 and 19,713 RFU/min/mL of culture. For Rgp, 93.5\% of total activity was associated with the bacteria and $6.5 \%$ was in the media, and for Kgp, 93.6\% was cell-associated and 6.4\% was in the media.

\section{Effect of sanggenol A on P. gingivalis gingipain activity}

Addition of Sanggenol A reduced the activity of pre-formed Rgp and Kgp gingipains. Results of (Table 1) shows that Sanggenol 
A had a dose-dependent inhibitory effect on Rgp activities of $P$. gingivalis cell-bound and culture media of the organism. In general, significantly ( $\mathrm{P}=0.0016$ ) higher degree of inhibition was seen in the cell-associated gingipains, compared to the activity of the cell culture media. However, Sanggenol A concentrations above $100 \mu \mathrm{M}$ had no significant difference in inhibition between activity of gingipains (Table 1) of cell-bound and culture media. Concentration of Sanggenol A above $100 \mu \mathrm{M}$ had no significant effect on the inhibition of Rgp gingipain activity (Table 1).

Table 1: Rgp gingipain activity of cell-bound and culture supernatant of $P$. gingivalis.

\begin{tabular}{|c|c|c|}
\hline $\boldsymbol{\mu M}$ of Sanggenol & $\begin{array}{c}\text { Supernatant } \\
\text { \% inhibition } \pm \text { SEM }\end{array}$ & $\begin{array}{c}\text { Cell-bound } \\
\text { \% inhibition } \pm \text { SEM }^{*}\end{array}$ \\
\hline 1 & $16 \pm 2$ & $52 \pm 2$ \\
\hline 3 & $34 \pm 3$ & $81 \pm 2$ \\
\hline 10 & $68 \pm 3$ & $91 \pm 4$ \\
\hline 30 & $92 \pm 12$ & $96 \pm 11$ \\
\hline 100 & $98 \pm 18$ & $98 \pm 12$ \\
\hline 300 & $99 \pm 21$ & $99 \pm 15$ \\
\hline 1000 & $100 \pm 17$ & $99 \pm 18$ \\
\hline
\end{tabular}

*=Standard error of mean

Rgp gingipain activity was calculated as percent inhibition of the control $\mathrm{RFU} / \mathrm{min} / \mathrm{ml}$ values of the control (in the absence of Sanggenol A).

Kgp gingipain activity was found to be more resistant to Sanggenol A (Table 2) compared to the Rgp gingipains. Sanggenol A up to $10 \mu \mathrm{M}$ had significantly less effect $(\mathrm{P}=0.0017)$ on Kgp gingipain activity than on Rgp gingipain. However, significant (P $<0.05$ ) inhibition of Kgp activity of cell-bound and culture media activities were seen with Sanggenol A concentrations of 3,000 and $10,000 \mu \mathrm{M}$ (Table 2). Also, to be noted that the inhibition of cell bound Kgp activity with $10,000 \mu \mathrm{M}$ of Sanggenol A was significantly different $(P=0.027)$ from inhibition of culture media activity. In general, our results indicate that the Rgp gingipain activity was relatively more sensitive to Sanggenol A than the Kgp activity.

Table 2: Effect of Sanggenol A on $P$. gingivalis Kgp gingipain activity of cell-bound and culture supernatant.

\begin{tabular}{|c|c|c|}
\hline & Supernatant & Cell-bound \\
\hline$\mu \mathrm{M}$ of Sanggenol & $\%$ inhibition \pm SEM $^{*}$ & $\%$ inhibition $\pm \mathrm{SEM}^{*}$ \\
\hline 1 & $9 \pm 3.0$ & $5 \pm 2$ \\
\hline 3 & $11 \pm 3$ & $7 \pm 3$ \\
\hline 10 & $14 \pm 2$ & $10 \pm 3$ \\
\hline 3000 & $35 \pm 9$ & $43 \pm 11$ \\
\hline 10,000 & $86 \pm 15$ & $69 \pm 13$ \\
\hline
\end{tabular}

*=Standard error of mean

Kgp gingipain activity was calculated as percent inhibition of the control $\mathrm{RFU} / \mathrm{min} / \mathrm{ml}$ values of the control (in the absence of Sanggenol A).

To further study the effect of Sanggenol A on the gingipain actiity of $P$. gingivalis, the bacteria were grown in the presence of 10 and $30 \mu \mathrm{M} / \mathrm{mL}$ Sanggenol A under similar conditions as described previously. Cells and culture media were collected and assayed for gingipain activity. The results (Table 3) show both Rgp activity of the cells and culture media was significantly reduced $(\mathrm{P}=0.0032)$ compared to the control cells grown without Sanggenol A. A 98\% inhibition of cell- associated Rgp activity was seen when the bacteria was grown in the presence of $30 \mu \mathrm{M}$ Sanggenol A. Cells grown in $30 \mu \mathrm{M}$ Sanggenol A had a significant inhibition $(\mathrm{P}=0.038)$ only on the secreted Kgp activity, but had no effect on the cell bound gingipain activity. Culturing bacteria with 10 and $30 \mu \mathrm{M}$ Sanggenol did not inhibit bacterial growth under these cultural conditions. As an additional control, we added Sanngenol A the end of the incubation, after the addition of TLCK, and found that Sanggenol A had no effect on fluorescence. This indicates that inhibition by Sanggenol A was not the result of fluorescence quenching.

Table 3: $P$. gingivalis Rgp and Kgp gingipain activity when the bacteria were grown in the presence of Sanggenol A.

\begin{tabular}{|c|c|c|c|c|}
\hline & \multicolumn{4}{|c|}{$\%$ inhibition of gingipain activity of: } \\
\hline $\boldsymbol{\mu M}$ Sanggenol A & Rgp \pm SEM$^{*}$ & & Kgp $\pm S E M$ & \\
\hline & Cell bound & $\begin{array}{c}\text { Culture } \\
\text { media }\end{array}$ & Cell bound & $\begin{array}{c}\text { Culture } \\
\text { media }\end{array}$ \\
\hline $10 \mu \mathrm{M}$ & $10 \pm 3$ & $18 \pm 2$ & Zero & $3 \pm 1$ \\
\hline $30 \mu \mathrm{M}$ & $98 \pm 11$ & $60 \pm 6$ & Zero & $15 \pm 2$ \\
\hline
\end{tabular}

* $=$ Standard error of mean

$\%$ inhibition was calculated based on the $\mathrm{P}$. gingivalis gingipain activity when the bacteria were grown in the absence of Sanggenol $A$ in the culture media.

\section{Discussion}

Prenylated flavonoids are a sub-class of naturally occurring plant-derived polyphenols whose structure combines a flavonoid backbone with a lipophilic prenyl group that is thought to enhance bioactivity by facilitating attachment and increasing uptake into cells [21]. These compounds are gaining importance due to a variety of biologic functions they possess, most notably their antioxidant, anti-inflammatory, anti-cancer, and anti-microbial properties [20]. The periodontal pathogen, P. gingivalis, has been known to induce destructive periodontal disease and is capable of evading host defenses. P. gingivalis was shown to cause periodontal destruction by secreting proteolytic enzymes Nakayama M, et al. [31] and interact with host cells in such a way that they secrete excessive inflammatory cytokines [32]. Gingipains, are a specific group of trypsin-like cysteine proteases produced by $P$. gingivalis that have been shown to degrade host proteins, promote bacterial adhesion, help evade phagocytosis, increase thrombin production, degrade fibrinogen, activate Kallikrein/Kinin system, promote MMP synthesis, impair neutrophil chemotaxis, and impair complement cascade [10]. A recent study by Dominy S, et al. [33], showed the evidence of presence of gingipains in the brain of Alzheimer's patients31.

In animal models, gingipain inhibition has been shown to exhibit marked reduction in virulence, decreased $P$. gingivalis CFUs, inhibition of lesions and decreased morbidity and fatality [3438]. Due to their role in pathogenicity of $P$. gingivalis, inhibition of gingipain activity may prove to be a potential therapeutic approach as an alternative to antibiotic therapy for attenuating the virulence of the periodontal pathogen and treatment or prevention of periodontal disease. Several classes of gingipain inhibitors have 
been identified, including plant-derived aromatic compounds called polyphenols, more specifically flavonoids [19]. Bodet C, et al. [39] showed the effectiveness in inhibiting proteolytic activity of P. gingivalis gingipains by a polyphenol component isolated from cranberry [39].

In our study, cell-associated gingipain activity was about 4.5 times more than secreted gingipain activity (for both Rgp and Kgp), and Rgp activity was about 4.5 times more than Kgp activity, which correlates with previous studies of gingipain [40-45]. It is very likely that due to the extensive post-translational modification of these proteases, most of the Rgp and Kgp may be bound to the Pg outer membrane [46]. Our results demonstrated the inhibitory effect of the prenylated flavonoid, Sanggenol A, on P. gingivalis gingipain activity. The inhibitory effect was more pronounced on pre-formed Rgp gingipain activity than the Kgp gingipain (Tables 1, 2). When grown with Pg, Sanggenol A also had a more inhibitory effect on cell bound and secreted Rgp activity (Table 3). The results appear to agree with the findings of other investigators who also reported greater Rgp gingipain inhibition $[21,47,48]$. There also appears to be some difference when comparing the effect of Sanggenol A on preformed gingipain activity with the gingipain activity seen after incubation with Sanggenol A. The exact mechanism of Sanngenol A inhibition is undetermined, but our results may indicate that Sanggenol A exhibits some degree of substantivity or inhibition of gingipains (specifically Rgp) as they are synthesized.

The differences between Rgp and Kgp susceptibility to inhibition seen with Sanggenol A and other inhibitors is interesting and draws attention to the differences between the two proteases. Rgp and Kgp the ability to selectively cleave different residues/peptides which are attributed to notable differences in the enzymes' molecular structure, specifically differences in active site configuration. While the mechanism of gingipain suppression in unclear, this difference in configuration/structure is likely to impact the differences in susceptibility to inhibition by Sanggenol A. There seems to be some disagreement in the literature as to which gingipain (Kgp or Rgp) is more important in Pg virulence [49-54]. Rgp and Kgp have been suggested to influence different aspects of $P$. gingivalis virulence. Rgp is primarily credited with activation of the kininkallikrein system and excessive bradykinin release, activation of clotting mechanism and over production of thrombin. Kgp interacts with (degrades) fibrinogen more specifically than Rgp prolonging clotting time [10].

In a murine model, Yoneda $\mathrm{M}$, et al. [49] showed that Rgpnull $P$. gingivalis produced significantly smaller lesions/less tissue destruction than Kgp-null P. gingivalis. While both Rgp and Kgp mutants produce smaller lesions than wild-type $P$ gingivalis, the Rgp-Kgp deficient $P$. gingivalis hardly induced lesions at all [49]. Along with these results, growing evidence suggest a synergistic relationship between Rgp and Kgp. An ideal therapeutic modality should target both Kgp and Rgp expression and secretion for the prevention and/or treatment of periodontitis and other P. gingivalisrelated infections. Our results provide evidence for inhibition of
P. gingivalis gingipain activity by Sanggenol A, suggesting that prenylated flavonoids may play a key role in reducing the pathogen's protease activity. Such an inhibition may help reduce not only the severity of periodontal disease but may also help treat systemic diseases associated with periodontitis. The effect of Sanggenol A on other periodontal pathogens also needs to be explored. Inhibitory effects of the prenylated flavonoids may have potential to develop new therapeutics for periodontal disease.

\section{Acknowledgements}

The study was partially funded by a grant from the University of Tennessee College of Dentistry Alumni Endowment Fund.

\section{Conflict of interest}

None.

\section{References}

1. Papapanou PN, Sanz M, Buduneli N, Dietrich T, Feres M, et al. (2018) Periodontitis: Consensus report of workgroup 2 of the 2017 World Workshop on the Classification of Periodontal and Peri-Implant Diseases and Conditions. J Periodontol 45 (Spec Iss): S162-S170.

2. Coats SR, Jones JW, Do CT, Braham PH, Bainbridge BW, et al. (2009) Human Toll-like receptor 4 responses to $P$. gingivalis are regulated by lipid A 1- and 4'- phosphatase activities. Cell Microbiol 11(11): 15871599.

3. Doungudomdacha S, Rawlinson A, Douglas CW (2000) Enumeration of Porphyromonas gingivalis, Prevotella intermedia and Actinobacillus actinomycetemcomitans in subgingival plaque samples by a quantitativecompetitive PCR method. J Med Microbiol 49(10): 861-874.

4. Kumar PS, Leyes EJ, Bryk JM, Martinez FJ, Moeschberger ML, et al. (2006) Changes in periodontal health status are associated with bacterial community shifts as assessed by quantitative $16 \mathrm{~S}$ cloning and sequencing. J. Clin. Microbiol 44(10): 3665-3673.

5. Darveau RP (2009) The oral microbial consortium's interaction with the periodontal innate defense system. DNA Cell Biol 28(8): 389-395.

6. Darveau RP (2010) Periodontitis: a polymicrobial disruption of host homeostasis. Nat Rev Microbiol 8(7): 481-490.

7. Hajishingallis G, Liang S, Payne M, Hashim A, Jotwani R, et al. (2011) A Low-Abundance Biofilm Species Orchestrates Inflammatory Periodontal Disease through the Commensal Microbiota and the Complement Pathway. Cell Host Microbe 10(5): 497-506.

8. Hajishengallis G, Lamont RJ (2012) Beyond the red complex and into more complexity: the polymicrobial synergy and dysbiosis (PSD) model of periodontal disease etiology. Mol Oral Microbial 27(6): 409-419.

9. Cheng WC, Saskia D, Burns LA, Evans HG, Walter GJ, et al. (2016) Periodontitis associated pathogens $P$. gingivalis and $A$. actinomycetemcomitans activate human CD14+ monocytes leading to enhanced Th17/IL-17 responses. Eur J Immunol 46(9): 2211-2221.

10. Imamura $\mathrm{T}$ (2003) The role of gingipains in the pathogenesis of periodontal disease. J Periodontol 74(1): 111-118.

11. Mysak J, Podzimek S, Sommerova P, Lyuya Mi Y, Bartova J, et al. (2014) Porphyromonas gingivalis: major periodontopathic pathogen overview. J Immunol Res 2014: 476068.

12. How KY, Song KP, Chan KG (2016) Porphyromonas gingivalis: an overview of periodontopathic pathogen below the gum line. Front Microbiol 7: 53.

13. Holt SC, Kesavalu L, Walker S, Genco CA (2000) Virulence factors of Porphyromonas gingivalis. Periodontol 20: 168-238.

14. Li N, Collyer CA (2011) Gingipains from Porphyromonas gingivalisComplex domain structures confer diverse functions. Eur J Microbiol Immunol 1(1): 41-58. 
15. Jayaprakash K, Khalaf H, Bengtsson T (2014) Gingipains from Porphyromonas gingivalis play a significant role in induction and regulation of CXCL8 in THP-1 cells. BMC Microbiology 14: 193.

16. Guo Y, Nguyen KA, Potempa J (2010) Dichotomy of gingipains action as virulence factors: from cleaving substrates with the precision of a surgeon's knife to a meat chopper-like brutal degradation of proteins. Periodontol 2000 54(1): 15-44.

17. Potempa J, Banbula A, Travis J (2000) Role of bacterial proteinases in matrix destruction and modulation of host responses. Periodontol 2000 24: 153-192.

18. Stathopoulou PG, Benakanakere MR, Galicia JC, Kinane DF (2009) The host cytokine response to Porphyromonas gingivalis is modified by gingipains. Oral Microbiol Immunol 24(1): 11-17.

19. Olsen I, Potempa J (2014) Strategies for the inhibition of gingipains for the potential treatment of periodontitis and associated systemic disease. J Oral Microbiol 6: 24800.

20. Kumar S, Pandey AK (2013) Chemistry and biological activities of flavonoids: an overview. ScientificWorldJournal 2013:162750.

21. Kariu T, Nakao R, Ikeda T, Nakashima K, Potempa J, et al. (2017) Inhibition of gingipains and Porphyromonas gingivalis growth and biofilm formation by prenyl flavonoids. J Periodontal Res 52(1): 89-96.

22. El Beshbishy HA, Singab AN, Sinkkonen J, Pihlaja K (2006) Hypolipidemic and antioxidant effects of Morus alba L. (Egyptian mulberry) root bark fractions supplementation in cholesterol-fed rats. Life Sci 78(23): 27242733.

23. Chung KO, Kim BY, Lee MH, Kim YR, Chung HY, et al. (2003) In-vitro and in-vivo anti-inflammatory effect of oxyresveratrol from Morus alba L. J Pharm Pharmacol 55(12): 1695-1700.

24. Kim AJ, Park S (2006) Mulberry extract supplements ameliorate the inflammation-related hematological parameters in carrageenaninduced arthritic rats. J Med Food 9(1): 431-435.

25. Zhang Z, Shi L (2010) Anti-inflammatory and analgesic properties of cismulberroside a from Ramulus mori. Fitoterapia 81(3): 214-218.

26. Khan MA, Rahman AA, Islam S, Khandokhar P, Parvin S, et al. (2013) A comparative study on the antioxidant activity of methanolic extracts from different parts of Morus alba L. (moraceae) BMC Res Notes 6: 2432 .

27. Park KM, You JS, Lee HY, Baek NI, Hwang JK (2003) Kuwanon G: an antibacterial agent from the root bark of Morus alba against oral pathogens. J Ethnopharmacol 84(2): 181-185.

28. Grienke U, Richter M, Walther E, Hoffmann A, Kirchmair J, et al. (2016) Discovery of prenylated flavonoids with dual activity against influenza virus and Streptococcus pneumoniae. Sci Rep 6: 27156.

29. Chen T, Nakayama K, Belliveau L, Duncan MJ (2001) Porphyromonas gingivalis and adhesion to epithelial cells. Infect Immun 69: 3048-3058.

30. Suwannakul S, Strafford GP, Whawell SA, Douglas CWI (2010) Identification of bistable populations of Porphyromonas gingivalis that differ in epithelial cell invasion. Microbiology 156: 3052-3064.

31. Nakayama M, Ohara N (2017) Molecular mechanisms of Porphyromonas gingivalis-host cell interaction on periodontal diseases. Jpn Dent Sci Rev 53(4): $134-140$.

32. Dominy S, Lynch C, Ermini F, Benedyk M, Marczyk A, et al. (2019) Porphyromonas gingivalis in Alzheimer's disease brains: Evidence for disease causation and treatment with small-molecule inhibitors. Sci Adv 5(1): eaau3333.

33. Dominy S, Lynch C, Ermini F, Benedyk M, Marczyk A, et al. (2019) Porphyromonas gingivalis in Alzheimer's disease brains: Evidence for disease causation and treatment with small-molecule inhibitors. Sci Adv 5(1): eaau3333.

34. Genco CA, Potempa J, Mikolajczyk Pawlinska J, Travis J (1999) Role of gingipains $\mathrm{R}$ in the pathogenesis of Porphyromonas gingivalis-mediated periodontal disease. Clin Infect Dis 28(3): 456-465.
35. Genco CA, Cutler CW, Kapczynski DR, Maloney KH, Arnold RR (1991) A novel mouse model to study the virulence of and host response to Porphyromonas (Bacteroides) gingivalis. Infect Immun 59(4): 12551263.

36. Genco CA, Arko RJ (1994) Animal chamber models for the study of hostparasite interactions. Bacterial pathogenesis, part A: identification and regulation of virulence factors. Methods Enzymol 235: 120-140.

37. Fletcher HM, Schenkein HA, Morgan RM, Bailey KA, Berry CR, et al. (1995) Virulence of a Porphyromonas gingivalis W83 mutant defective in the prtH gene. Infect Immun 63(4): 1521-1528.

38. Nakayama KM, Yoshimura F, Kadowaki T, Yamamoto K (1996) Involvement of arginine-specific cysteine proteinases (arg-gingipain) in fimbriation of Porphyromonas gingivalis. J Bacteriol 178: 2818-2824.

39. Bodet C, Piche M, Chandad F, Grenier D. (2006) Inhibition of periodontopathogen-derived proteolytic enzymes by a high-molecularweight fraction isolated from cranberry. J Antimicrob Chemother 57(4): 685-690.

40. Takii R, Kadowaki T, Baba A, Tsukuba T, Yamamoto K (2005) A functional virulence complex composed of gingipains, adhesins, and lipopolysaccharides shows high affinity to host cells and matrix proteins and escapes recognition by host immune systems. Infect Immun 73(2): 883-893.

41. Nguyen K, Potempa J (2013) Gingipain R. In: Barrett A, Rawlings ND, Woessner J (eds) Handbook of Proteolytic Enzymes. Waltham Academic Press 2013: 2329-2336.

42. Potempa J, Pike R, Travis J (1995) The multiple forms of trypsin-like activity present in various strains of Porphyromonas gingivalis are due to the presence of either Arg-gingipain or Lys-gingipain. Infect Immun 63(4): 1176-1182.

43. Potempa J, Banbula A, Travis J (2000) Role of bacterial proteinases in matrix destruction and modulation of host responses. Periodontol 2000 24: 153-192.

44. Potempa J, Sroka A, Imamura T, Travis J (2003) Gingipains, the major cysteine proteinases and virulence factors of Porphyromonas gingivalis: structure, function and assembly of multidomain protein complexes. Curr Protein Pept Sci 4(6): 397-407.

45. Hosn KN, Jefferson MM, Carlton L, Solomon SA, Thomas EL (2015) Inhibitors of Bacterial Protease Enzymes for Periodontal Therapy. Clin Exp Dent Res 1: 18-25.

46. Zhou XY, Gao JL, Hunter N, Potempa J, Nguyen KA (2013) Sequenceindependent processing site of the C-terminal domain (CTD) influences maturation of the RgpB protease from Porphyromonas gingivalis. Mol Microbiol 89(5): 903-917.

47. Singh AK, Ydav S, Sharma K (2018) Quantum curcumin mediated inhibition of gingipains and mixed-biofilm of Porphyromonas gingivalis causing chronic periodontitis. RSC Advances 8: 40426-40445.

48. Genco C, Potempa J, Mikolajczyk-Pawlinska J, Travis J (1999) Role of gingipains $\mathrm{R}$ in the pathogenesis of Porphyromonas gingivalis-mediated periodontal disease. Clin Infect Dis 28(3): 456-465.

49. Yoneda M, Hirofuju T, Anan H (2001) Mixed infection of Porphyromonas gingivalis and Bacteriodes forsythus in a murine abscess model. J Periodontal Res 36(4): 237-243.

50. Nakayama K, Kadowaki T, Okamoto K, Yamamoto K (1995) Construction and characterization of arginine-specific cysteine proteinase (Arggingipain)-deficient mutants of Porphyromonas gingivalis, Evidence for significant contribution of Arg-gingipain to virulence. J Biol Chem 270(40): 23619-23626.

51. Wilensky A, Polak D, Houri Haddad Y, Shapira L (2013) The role of RgpA in the pathogenicity of Porphyromonas gingivalis in the murine periodontitis model. J Clin Periodontol 40: 924-932.

52. Mezyk Kopec R, Bzowska M, Potempa J, Bzowska M, Jura N, et al. (2005) Inactivation of membrane tumor necrosis factor alpha by gingipains from Porphyromonas gingivalis. Infect Immun 73(3): 1506-1514. 
53. Pathirana RD, O'BrienSimpson NM, Brammar GC, Slakeski N, Reynolds EC (2007) Kgp and RgpB, but not RgpA, are important for Porphyromonas gingivalis virulence in the murine periodontitis model. Infect Immun 75(3): 1436-1442.
54. Curtis MA, Aduse Opoku J, Rangarajan M, Gallagher A, Sterne JA, et al. (2002) Attenuation of the virulence of Porphyromonas gingivalis by using a specific synthetic Kgp protease inhibitor. Infect Immun 70(12): 6968-6975. 